\title{
Contents Tables
}

SIFigure 1 Optimized geometries of isomers, reactants, products and interconversion transition states for the title reaction at the B3LYP/6-31G(d) level. Bond lengths are in angstroms and angles in degrees.

SITable 1 Total (a. u.) and relative energies in parentheses (kcal/mol) of the reactants, intermediate isomers, products and transition states at the G3B3 level. The italic values are at the CBS-QB3 level. 
SIFigure 1
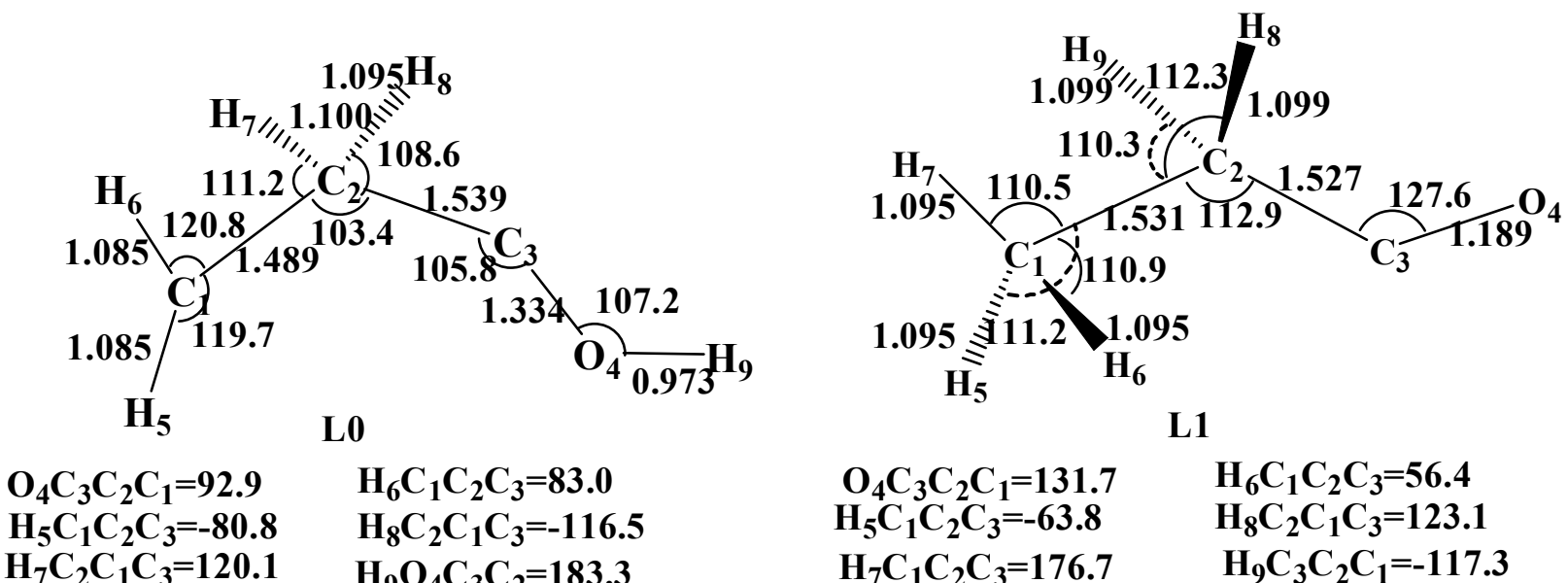

$\mathrm{H}_{7} \mathrm{C}_{2} \mathrm{C}_{1} \mathrm{C}_{3}=120.1$

$\mathrm{H}_{9} \mathrm{O}_{4} \mathrm{C}_{3} \mathrm{C}_{2}=183.3$

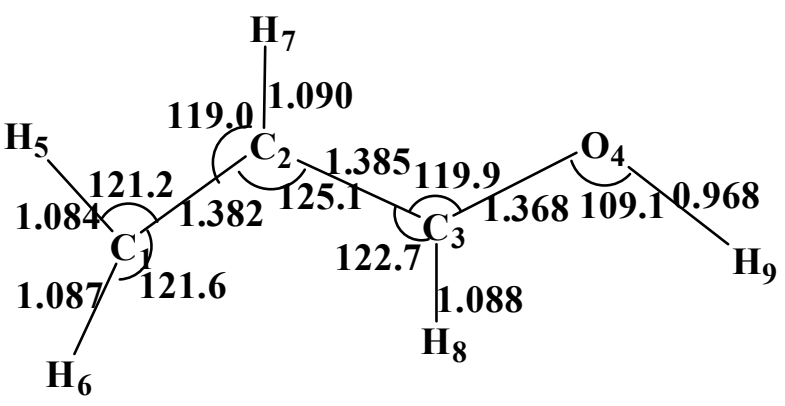

L2(Cs)
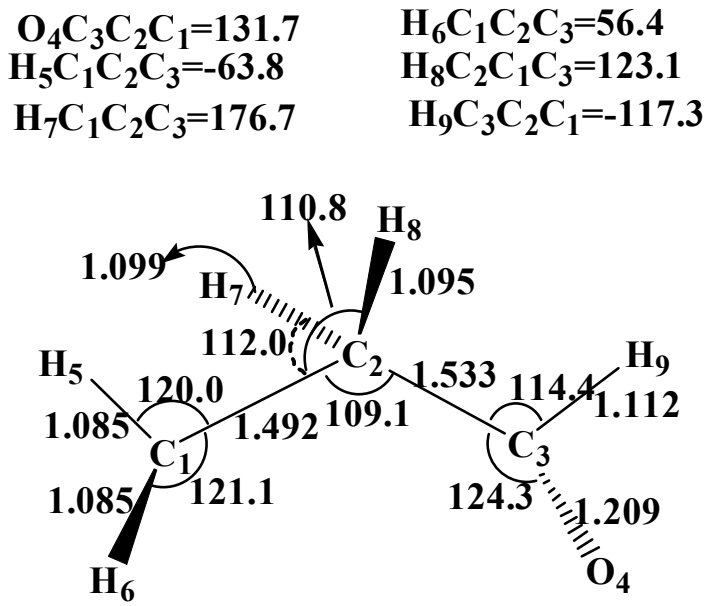

L3

$\mathrm{O}_{4} \mathrm{C}_{3} \mathrm{C}_{2} \mathrm{C}_{1}=110.8 \quad \mathrm{H}_{6} \mathrm{C}_{1} \mathrm{C}_{2} \mathrm{C}_{3}=-81.9$

$\mathrm{H}_{5} \mathrm{C}_{1} \mathrm{C}_{2} \mathrm{C}_{3}=86.0 \quad \mathrm{H}_{8} \mathrm{C}_{2} \mathrm{C}_{1} \mathrm{C}_{3}=-118.8$

$\mathrm{H}_{7} \mathrm{C}_{2} \mathrm{C}_{1} \mathrm{C}_{3}=118.8 \quad \mathrm{H}_{9} \mathrm{C}_{3} \mathrm{C}_{2} \mathrm{C}_{1}=-66.8$

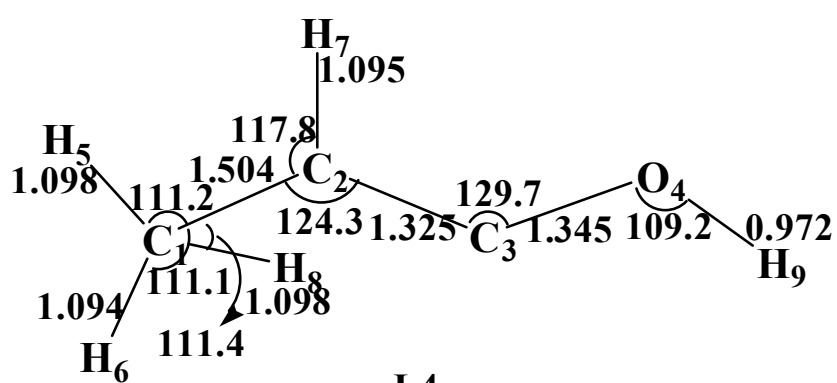

L4

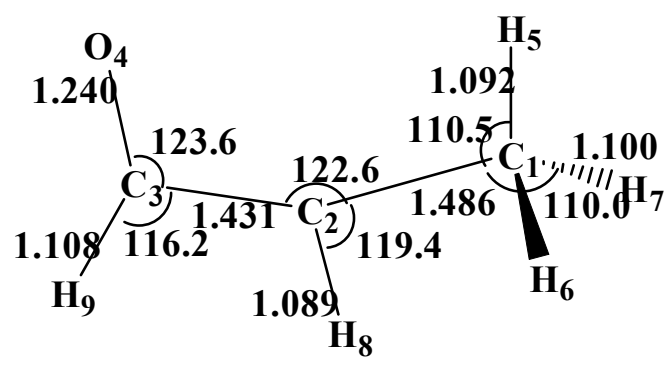

$\mathrm{O}_{4} \mathrm{C}_{3} \mathrm{C}_{2} \mathrm{C}_{1}=170.4 \quad \mathrm{H}_{6} \mathrm{C}_{1} \mathrm{C}_{2} \mathrm{C}_{3}=-\mathbf{0 . 4}$

$\mathrm{H}_{5} \mathrm{C}_{1} \mathrm{C}_{2} \mathrm{C}_{3}=-120.7 \quad \mathrm{H}_{8} \mathrm{C}_{1} \mathrm{C}_{2} \mathrm{C}_{3}=-239.8$

L5 (Cs)

$\mathrm{H}_{7} \mathrm{C}_{2} \mathrm{C}_{1} \mathrm{C}_{3}=-178.6 \quad \mathrm{H}_{9} \mathrm{O}_{4} \mathrm{C}_{3} \mathrm{C}_{2}=135.3$

$\mathrm{H}_{6} \mathrm{C}_{1} \mathrm{C}_{2} \mathrm{C}_{3}=121.2 \quad \mathrm{H}_{7} \mathrm{C}_{1} \mathrm{C}_{2} \mathrm{C}_{3}=-121.2$ 
SIFigure 1 (Continued)

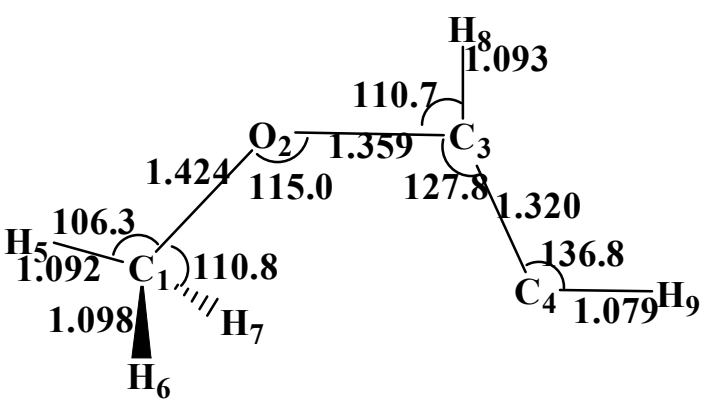

L6 (Cs)

$\mathrm{H}_{6} \mathrm{C}_{1} \mathrm{O}_{2} \mathrm{C}_{3}=-60.4 \quad \mathrm{H}_{7} \mathrm{C}_{1} \mathrm{O}_{2} \mathrm{C}_{3}=60.4$

$\mathrm{H}_{7}$

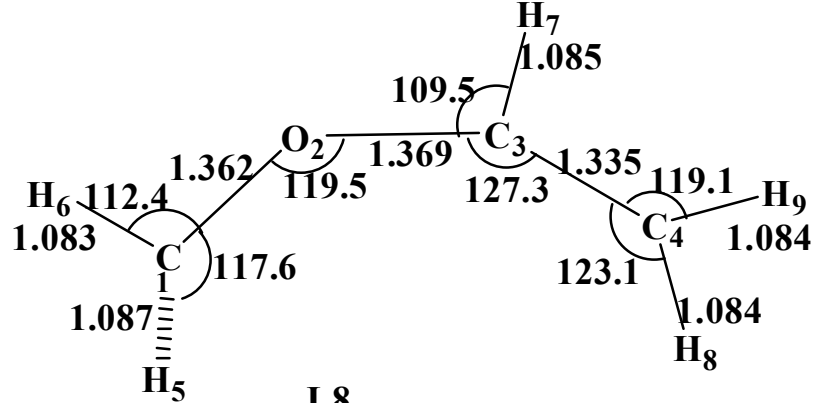

L8

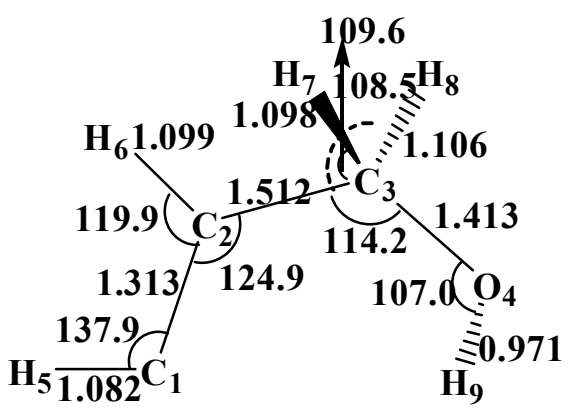

L7

$\mathrm{H}_{5} \mathrm{C}_{1} \mathrm{C}_{2} \mathrm{C}_{3}=-179.5 \quad \mathrm{H}_{8} \mathrm{C}_{3} \mathrm{C}_{2} \mathrm{C}_{1}=125.7$

$\mathrm{H}_{7} \mathrm{C}_{2} \mathrm{C}_{1} \mathrm{C}_{3}=-118.6 \quad \mathrm{H}_{9} \mathrm{O}_{4} \mathrm{C}_{3} \mathrm{C}_{2}=45.8$

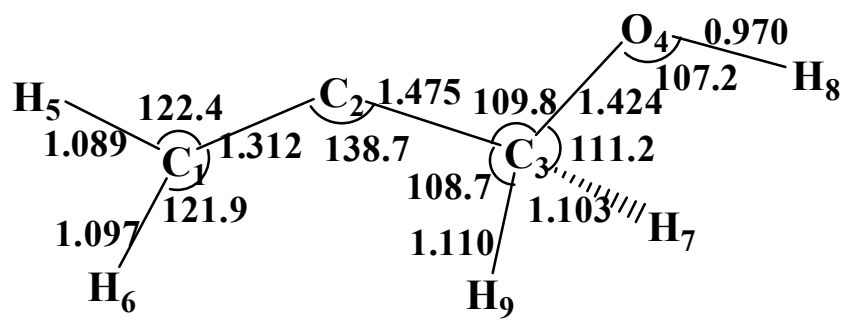

L9

$\mathrm{O}_{4} \mathrm{C}_{3} \mathrm{C}_{2} \mathrm{C}_{1}=232.6$

$\mathrm{H}_{5} \mathrm{C}_{1} \mathrm{C}_{2} \mathrm{C}_{3}=-176.4 \quad \mathrm{H}_{8} \mathrm{O}_{4} \mathrm{C}_{3} \mathrm{C}_{2}=-176.4$

$\mathrm{H}_{7} \mathrm{C}_{3} \mathrm{C}_{2} \mathrm{C}_{1}=110.6 \quad \mathrm{H}_{9} \mathrm{C}_{3} \mathrm{C}_{2} \mathrm{C}_{1}=-5.5$

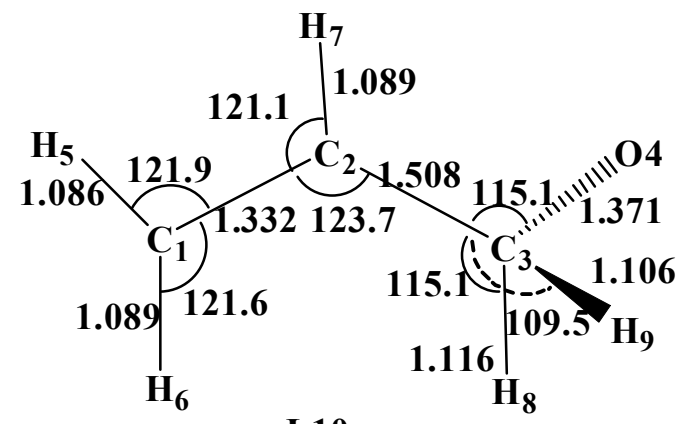

L10

$\mathrm{O}_{4} \mathrm{C}_{3} \mathrm{C}_{2} \mathrm{C}_{1}=135.4$

$\mathrm{H}_{6} \mathrm{C}_{1} \mathrm{C}_{2} \mathrm{C}_{3}=-0.6$

$\mathrm{H}_{5} \mathrm{C}_{1} \mathrm{C}_{2} \mathrm{C}_{3}=179.4$

$\mathrm{H}_{8} \mathrm{C}_{3} \mathrm{C}_{2} \mathrm{C}_{1}=135.4$

$\mathrm{H}_{7} \mathrm{C}_{2} \mathrm{C}_{1} \mathrm{C}_{3}=179.8$

$\mathrm{H}_{9} \mathrm{C}_{3} \mathrm{C}_{2} \mathrm{C}_{1}=-109.4$ $\mathrm{H}_{8 / 1 / 1 / 2}^{1.089} \mathbf{1 . 0 9 0}^{\mathrm{H}_{7}}$

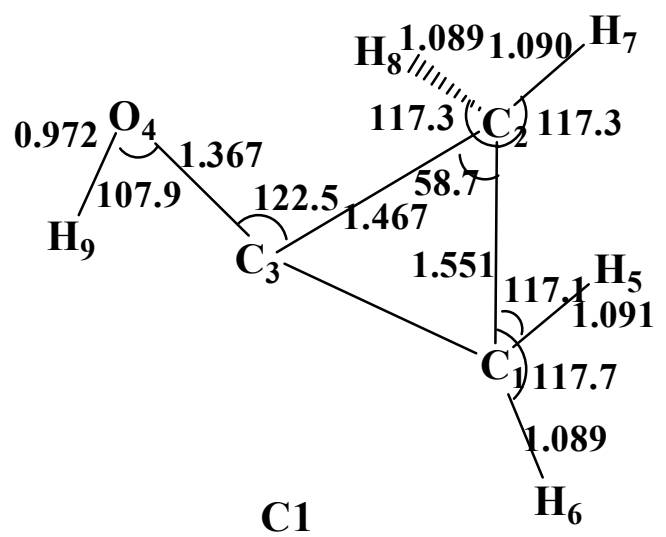

$\mathrm{O}_{4} \mathrm{C}_{3} \mathrm{C}_{2} \mathrm{C}_{1}=117.6 \quad \mathrm{H}_{6} \mathrm{C}_{1} \mathrm{C}_{2} \mathrm{C}_{3}=109.2$

$\mathrm{H}_{5} \mathrm{C}_{1} \mathrm{C}_{2} \mathrm{C}_{3}=-108.6 \quad \mathrm{H}_{8} \mathrm{C}_{2} \mathrm{C}_{1} \mathrm{C}_{3}=-109.5$

$\mathrm{H}_{7} \mathrm{C}_{2} \mathrm{C}_{1} \mathrm{C}_{3}=108.4 \quad \mathrm{H}_{9} \mathrm{O}_{4} \mathrm{C}_{3} \mathrm{C}_{2}=215.3$ 
SIFigure 1 (Continued)

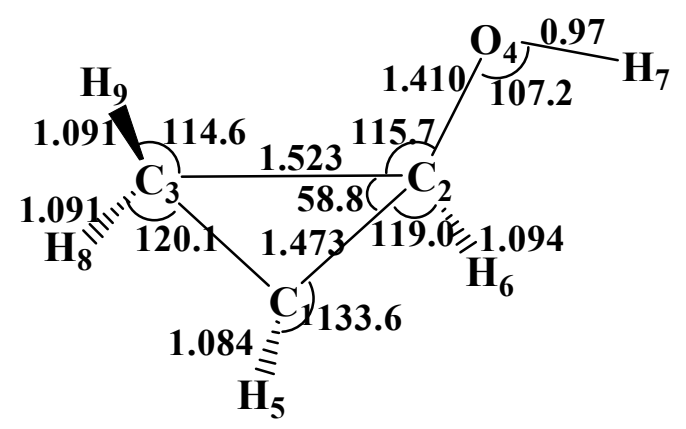

\section{C2}

$\mathrm{O}_{4} \mathrm{C}_{2} \mathrm{C}_{3} \mathrm{C}_{1}=-111.1 \quad \mathrm{H}_{6} \mathrm{C}_{2} \mathrm{C}_{1} \mathrm{C}_{3}=124.3$

$\mathrm{H}_{5} \mathrm{C}_{1} \mathrm{C}_{2} \mathrm{C}_{3}=124.3 \quad \mathrm{H}_{8} \mathrm{C}_{3} \mathrm{C}_{2} \mathrm{C}_{1}=-109.1$

$\mathrm{H}_{7} \mathrm{C}_{4} \mathrm{C}_{2} \mathrm{C}_{1}=62.3 \quad \mathrm{H}_{9} \mathrm{C}_{3} \mathrm{C}_{2} \mathrm{C}_{1}=109.3$

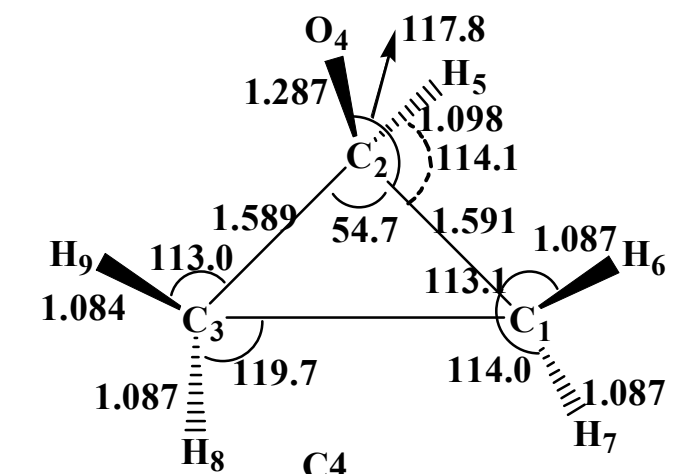

$\mathrm{O}_{4} \mathrm{C}_{3} \mathrm{C}_{2} \mathrm{C}_{1}=105.9$

$\mathrm{H}_{5} \mathrm{C}_{2} \mathrm{C}_{1} \mathrm{C}_{3}=-103.5$

$\mathrm{H}_{6} \mathrm{C}_{1} \mathrm{C}_{2} \mathrm{C}_{3}=-112.0$

$\mathrm{H}_{7} \mathrm{C}_{1} \mathrm{C}_{2} \mathrm{C}_{3}=112.4$

$\mathrm{H}_{8} \mathrm{C}_{3} \mathrm{C}_{1} \mathrm{C}_{2}=103.5$

$\mathrm{H}_{9} \mathrm{C}_{3} \mathrm{C}_{2} \mathrm{C}_{1}=112.0$

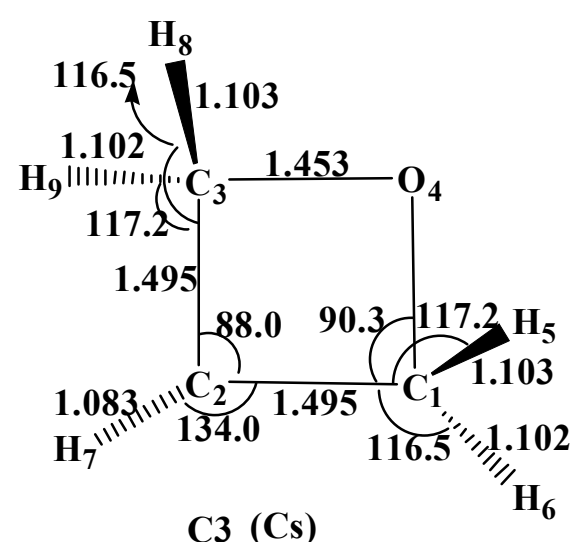

$\mathrm{O}_{4} \mathrm{C}_{1} \mathrm{C}_{2} \mathrm{C}_{3}=-\mathbf{1 . 6}$

$\mathrm{H}_{6} \mathrm{C}_{1} \mathrm{C}_{2} \mathrm{C}_{3}=-116.5$

$\mathrm{H}_{5} \mathrm{C}_{1} \mathrm{C}_{2} \mathrm{C}_{3}=113.4 \quad \mathrm{H}_{8} \mathrm{C}_{3} \mathrm{C}_{2} \mathrm{C}_{1}=-113.4$

$\mathrm{H}_{7} \mathrm{C}_{2} \mathrm{C}_{1} \mathrm{C}_{4}=-160.6$

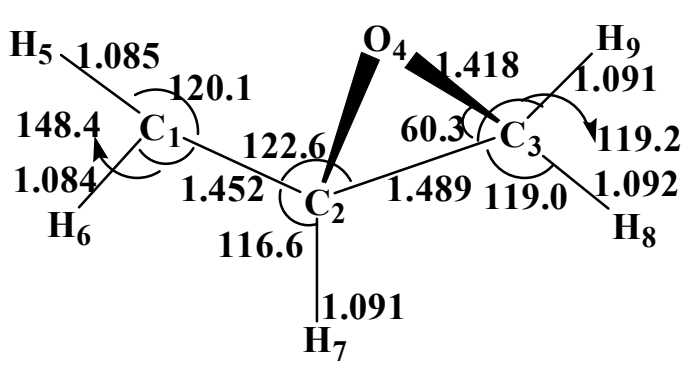

$\begin{array}{ll}\mathrm{O}_{4} \mathrm{C}_{3} \mathrm{C}_{2} \mathrm{C}_{1}=103.3 & \mathrm{C} 5 \\ \mathrm{H}_{5} \mathrm{C}_{1} \mathrm{C}_{2} \mathrm{C}_{3}=-32.5 & \mathrm{H}_{6} \mathrm{C}_{1} \mathrm{C}_{2} \mathrm{C}_{3}=148.4 \\ \mathrm{H}_{7} \mathrm{C}_{2} \mathrm{C}_{1} \mathrm{C}_{3}=-154.8 & \mathrm{H}_{8} \mathrm{C}_{3} \mathrm{C}_{2} \mathrm{C}_{1}=-151.5 \\ \mathrm{H}_{9} \mathrm{C}_{3} \mathrm{C}_{2} \mathrm{C}_{1}=-1.5\end{array}$

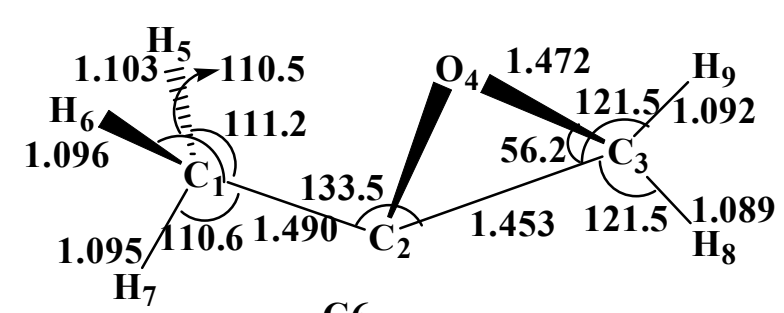

$\mathrm{O}_{4} \mathrm{C}_{3} \mathrm{C}_{2} \mathrm{C}_{1}=110.4$

$\mathrm{H}_{5} \mathrm{C}_{1} \mathrm{C}_{2} \mathrm{C}_{3}=-29.0$

$\mathrm{H}_{7} \mathrm{C}_{1} \mathrm{C}_{2} \mathrm{C}_{3}=91.4$
C6

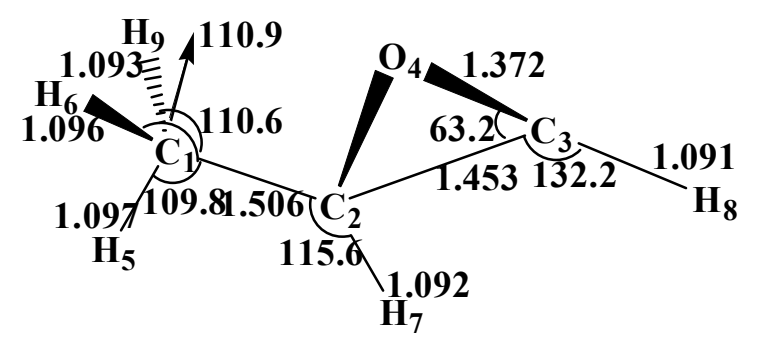

C7

$\mathrm{O}_{4} \mathrm{C}_{3} \mathrm{C}_{2} \mathrm{C}_{1}=102.0$

$\mathrm{H}_{5} \mathrm{C}_{1} \mathrm{C}_{2} \mathrm{C}_{3}=99.9$

$\mathrm{H}_{7} \mathrm{C}_{2} \mathrm{C}_{1} \mathrm{C}_{3}=-161.5$
$\mathrm{H}_{6} \mathrm{C}_{1} \mathrm{C}_{2} \mathrm{C}_{3}=-140.8$

$\mathrm{H}_{8} \mathrm{C}_{3} \mathrm{C}_{2} \mathrm{C}_{1}=-149.1$

$\mathrm{H}_{9} \mathrm{C}_{1} \mathrm{C}_{2} \mathrm{C}_{3}=-19.9$ 
SIFigure 1 (Continued)
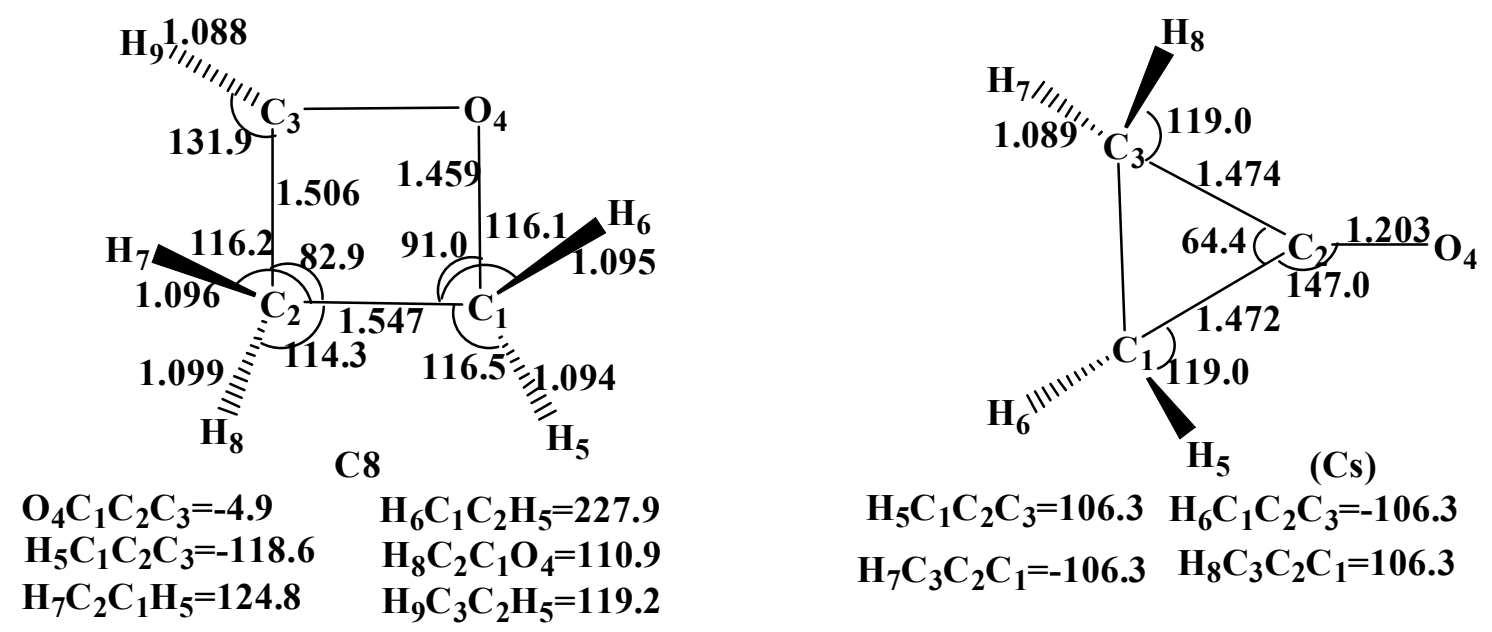

$\mathrm{H}_{5} \mathrm{C}_{1} \mathrm{C}_{2} \mathrm{C}_{3}=106.3 \quad \mathrm{H}_{6} \mathrm{C}_{1} \mathrm{C}_{2} \mathrm{C}_{3}=-106.3$

$\mathrm{H}_{7} \mathrm{C}_{3} \mathrm{C}_{2} \mathrm{C}_{1}=-106.3 \quad \mathrm{H}_{8} \mathrm{C}_{3} \mathrm{C}_{2} \mathrm{C}_{1}=106.3$
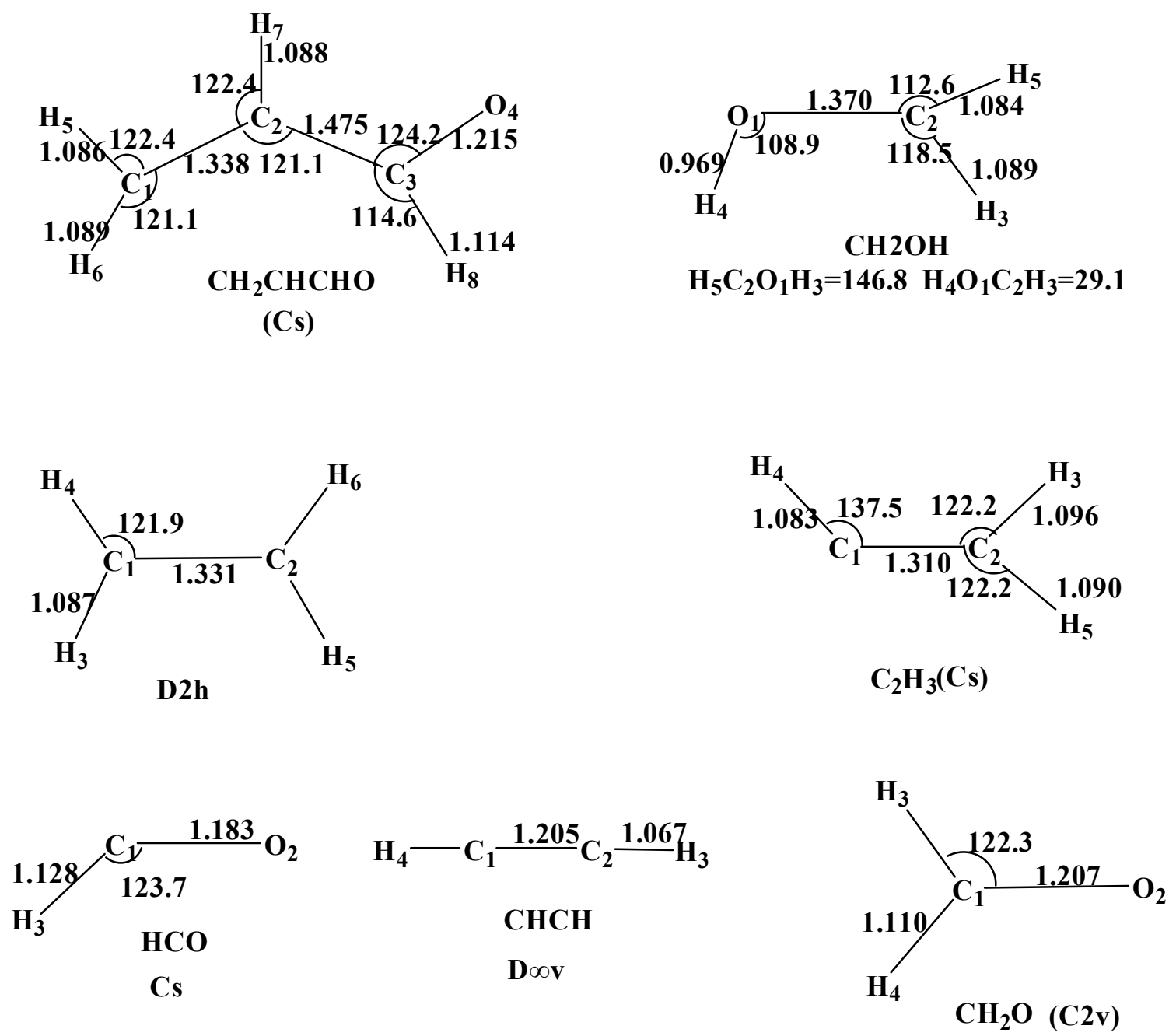


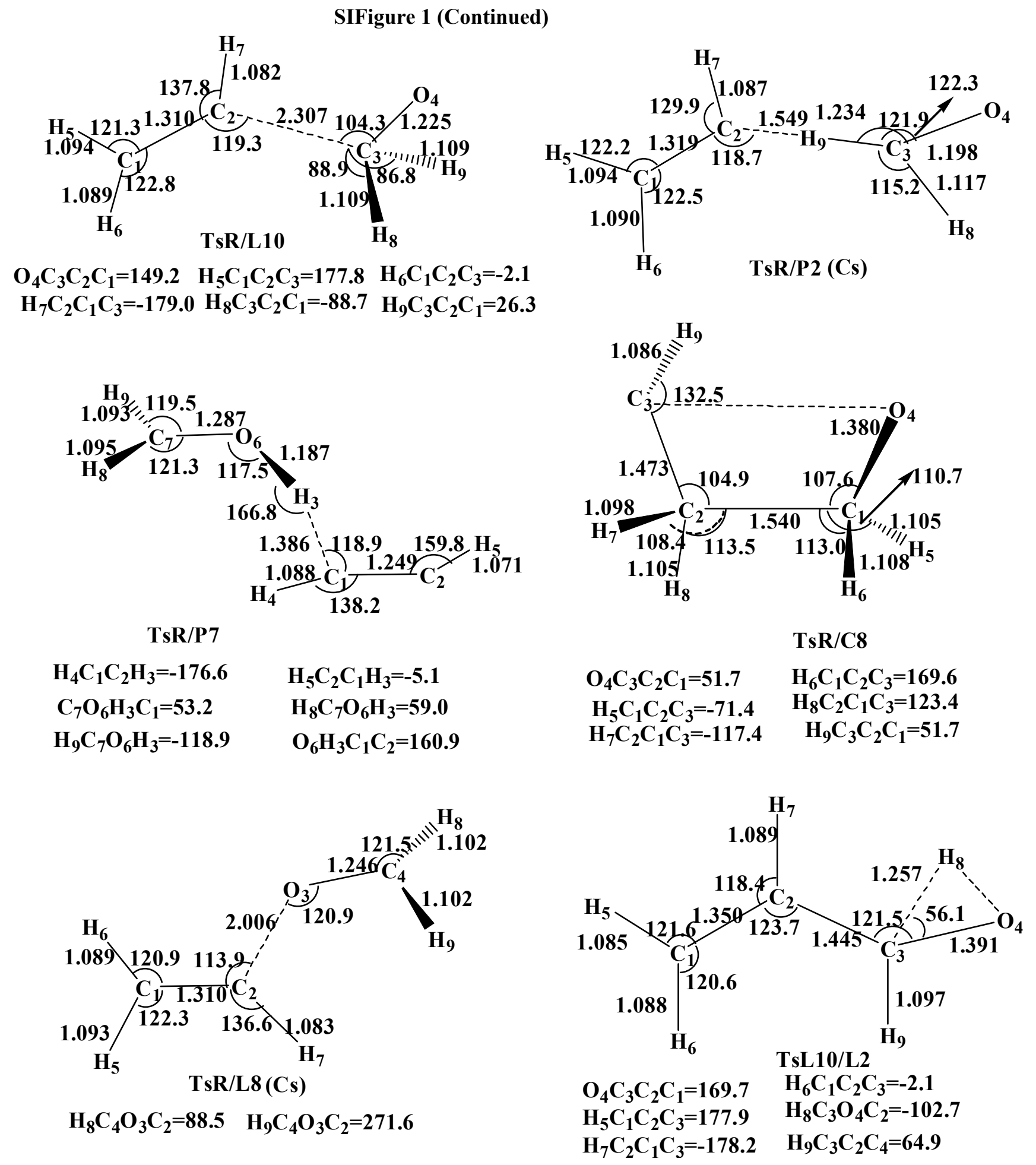


SIFigure 1 (Continued)

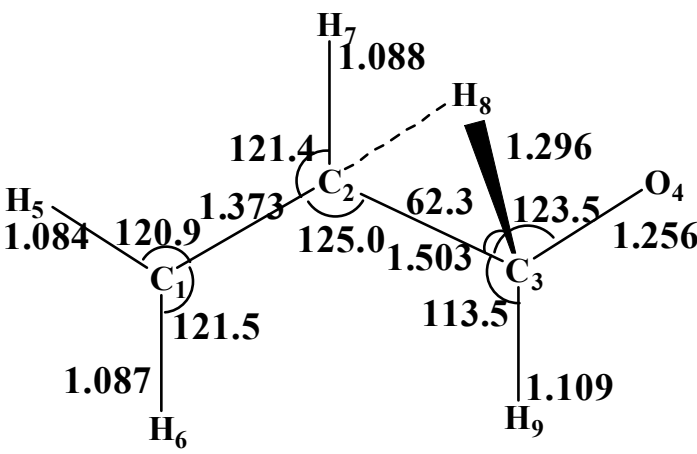

TsL10/L3

$$
\begin{array}{ll}
\mathrm{O}_{4} \mathrm{C}_{3} \mathrm{C}_{2} \mathrm{C}_{1}=167.5 & \mathrm{H}_{6} \mathrm{C}_{1} \mathrm{C}_{2} \mathrm{C}_{3}=0.6 \\
\mathrm{H}_{5} \mathrm{C}_{1} \mathrm{C}_{2} \mathrm{C}_{3}=-179.6 & \mathrm{H}_{8} \mathrm{C}_{3} \mathrm{C}_{2} \mathrm{C}_{1}=-93.0 \\
\mathrm{H}_{7} \mathrm{C}_{2} \mathrm{C}_{1} \mathrm{C}_{3}=178.4 & \mathrm{H}_{9} \mathrm{C}_{3} \mathrm{C}_{2} \mathrm{C}_{4}=-0.5
\end{array}
$$

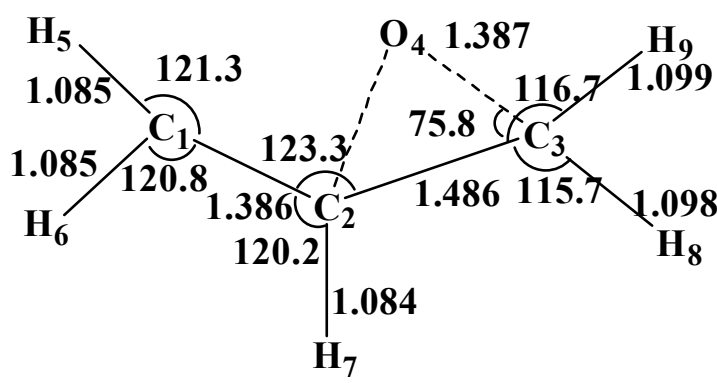

TsL10/C5

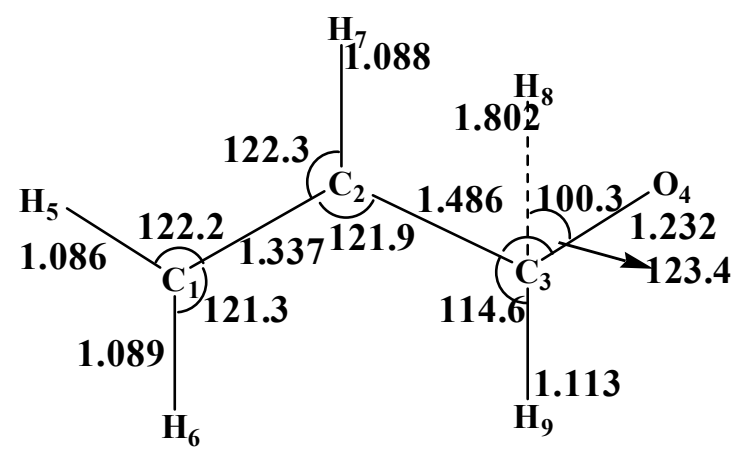

TsL10/P1

$\mathrm{O}_{4} \mathrm{C}_{3} \mathrm{C}_{2} \mathrm{C}_{1}=166.9 \quad \mathrm{H}_{6} \mathrm{C}_{1} \mathrm{C}_{2} \mathrm{C}_{3}=2.2$

$\mathrm{H}_{5} \mathrm{C}_{1} \mathrm{C}_{2} \mathrm{C}_{3}=-177.6 \quad \mathrm{H}_{8} \mathrm{C}_{3} \mathrm{O}_{4} \mathrm{C}_{2}=-102.4$

$\mathrm{H}_{7} \mathrm{C}_{2} \mathrm{C}_{1} \mathrm{C}_{3}=177.6 \quad \mathrm{H}_{9} \mathrm{C}_{3} \mathrm{C}_{2} \mathrm{C}_{4}=-0.5$

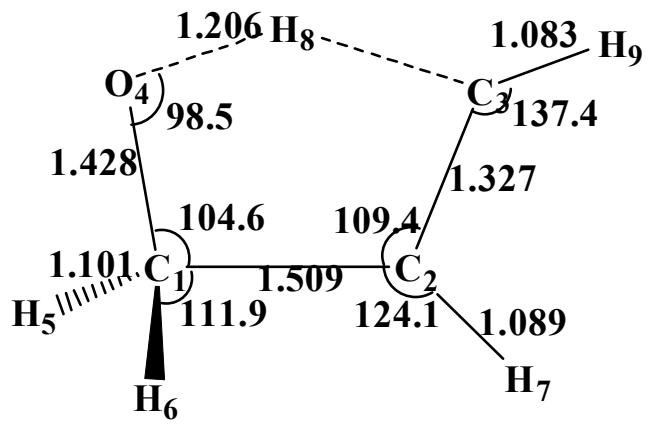

TsL10/L(Cs)

$\mathrm{H}_{5} \mathrm{C}_{1} \mathrm{C}_{2} \mathrm{C}_{3}=-119.4 \quad \mathrm{H}_{6} \mathrm{C}_{1} \mathrm{C}_{2} \mathrm{C}_{3}=119.4$

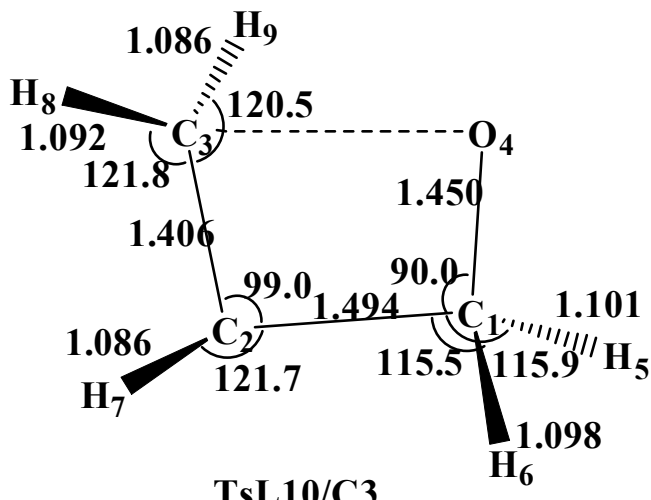

TsL10/C3

$$
\begin{array}{cc}
\mathrm{O}_{4} \mathrm{C}_{3} \mathrm{C}_{2} \mathrm{C}_{1}=33.1 & \mathrm{H}_{6} \mathrm{C}_{1} \mathrm{C}_{2} \mathrm{C}_{3}=149.1 \\
\mathrm{H}_{5} \mathrm{C}_{1} \mathrm{C}_{2} \mathrm{C}_{3}=-81.2 & \mathrm{H}_{8} \mathrm{C}_{3} \mathrm{C}_{2} \mathrm{C}_{1}=-144.4 \\
\mathrm{H}_{7} \mathrm{C}_{2} \mathrm{C}_{1} \mathrm{C}_{3}=-135.7 & \mathrm{H}_{9} \mathrm{C}_{3} \mathrm{C}_{2} \mathrm{C}_{1}=57.6
\end{array}
$$

$$
\begin{array}{ll}
\mathrm{O}_{4} \mathrm{C}_{3} \mathrm{C}_{2} \mathrm{C}_{1}=145.2 & \mathrm{H}_{6} \mathrm{C}_{1} \mathrm{C}_{2} \mathrm{C}_{3}=-71.3 \\
\mathrm{H}_{5} \mathrm{C}_{1} \mathrm{C}_{2} \mathrm{C}_{3}=143.8 & \mathrm{H}_{8} \mathrm{C}_{3} \mathrm{C}_{2} \mathrm{C}_{1}=-135.5 \\
\mathrm{H}_{7} \mathrm{C}_{2} \mathrm{C}_{1} \mathrm{C}_{3}=-177.9 & \mathrm{H}_{9} \mathrm{C}_{3} \mathrm{C}_{2} \mathrm{C}_{1}=-0.3
\end{array}
$$


SIFigure 1 (Continued)
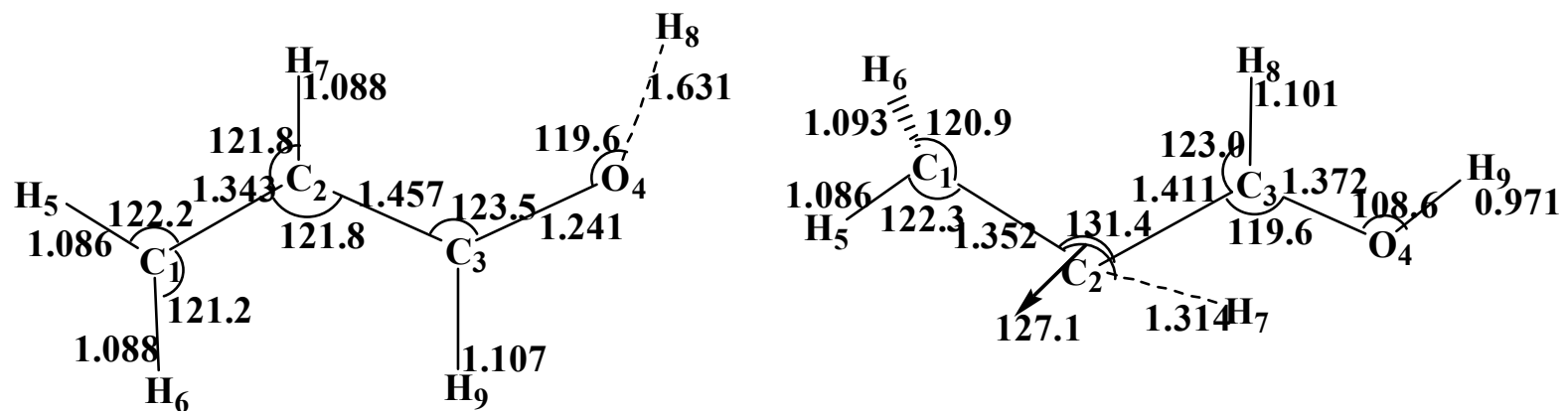

TsL2/P1

$$
\begin{array}{ll}
\mathrm{O}_{4} \mathrm{C}_{2} \mathrm{C}_{1} \mathrm{C}_{3}=177.8 & \mathrm{H}_{6} \mathrm{C}_{1} \mathrm{C}_{2} \mathrm{C}_{3}=1.0 \\
\mathrm{H}_{5} \mathrm{C}_{1} \mathrm{C}_{2} \mathrm{C}_{3}=-178.7 & \mathrm{H}_{8} \mathrm{C}_{3} \mathrm{C}_{2} \mathrm{C}_{1}=102.4 \\
\mathrm{H}_{7} \mathrm{C}_{2} \mathrm{C}_{1} \mathrm{C}_{3}=178.8 & \mathrm{H}_{9} \mathrm{O}_{4} \mathrm{C}_{3} \mathrm{C}_{2}=-3.0
\end{array}
$$

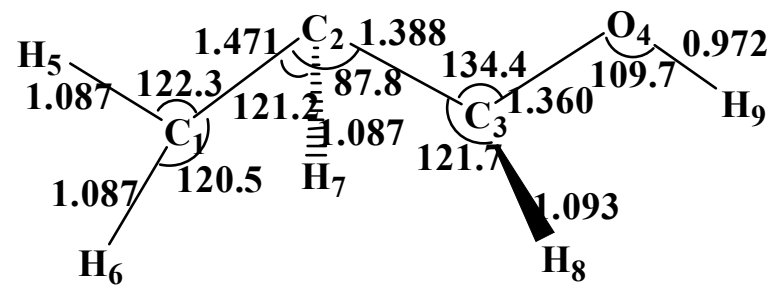

TsL2/C2

$\mathrm{O}_{4} \mathrm{C}_{2} \mathrm{C}_{1} \mathrm{C}_{3}=-98.3 \quad \mathrm{H}_{6} \mathrm{C}_{1} \mathrm{C}_{2} \mathrm{C}_{3}=-102.8$

$\mathrm{H}_{5} \mathrm{C}_{1} \mathrm{C}_{2} \mathrm{C}_{3}=80.4 \quad \mathrm{H}_{8} \mathrm{C}_{3} \mathrm{C}_{2} \mathrm{C}_{1}=-64.0$

$\mathrm{H}_{7} \mathrm{C}_{2} \mathrm{C}_{1} \mathrm{C}_{3}=123.4 \quad \mathrm{H}_{9} \mathrm{O}_{4} \mathrm{C}_{3} \mathrm{C}_{2}=-178.6$
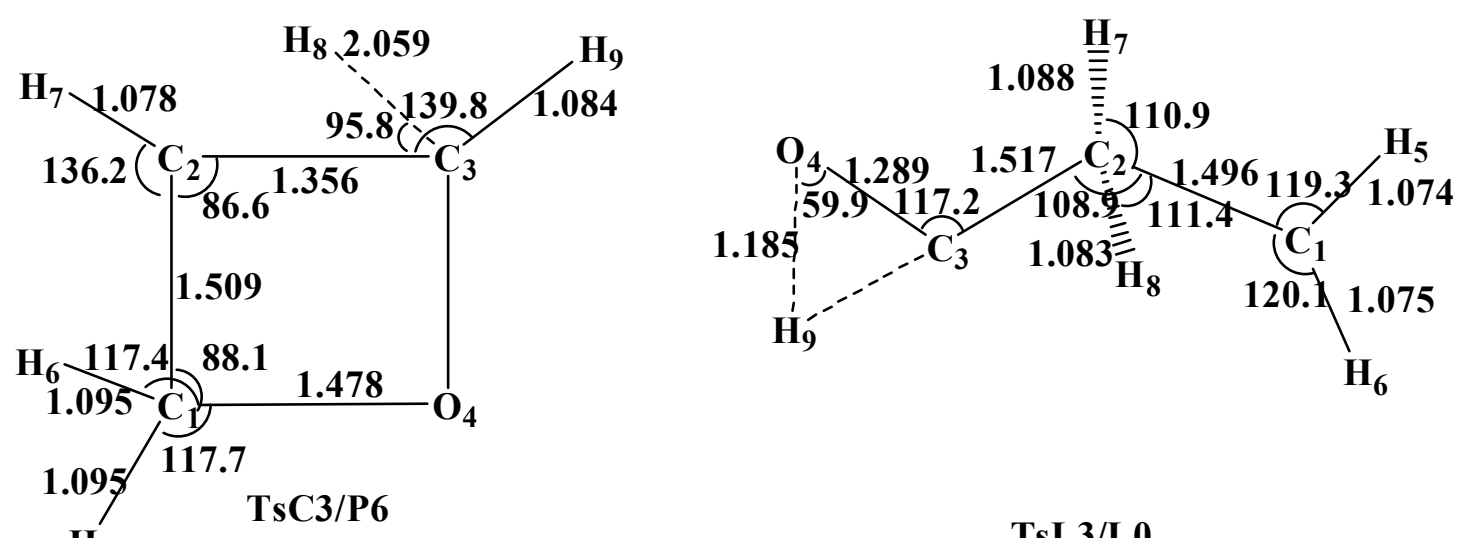

$\mathrm{O}_{4} \mathrm{C}_{2} \mathrm{C}_{1} \mathrm{C}_{3}=207.7 \quad \mathrm{H}_{6} \mathrm{C}_{1} \mathrm{C}_{2} \mathrm{C}_{3}=37.4$

$\mathrm{H}_{5} \mathrm{C}_{1} \mathrm{C}_{2} \mathrm{C}_{3}=-148.1 \quad \mathrm{H}_{8} \mathrm{C}_{3} \mathrm{C}_{2} \mathrm{C}_{1}=12.0$

$\mathrm{H}_{7} \mathrm{C}_{2} \mathrm{C}_{1} \mathrm{C}_{3}=78.8 \quad \mathrm{H}_{9} \mathrm{O}_{4} \mathrm{C}_{3} \mathrm{C}_{2}=-161.6$

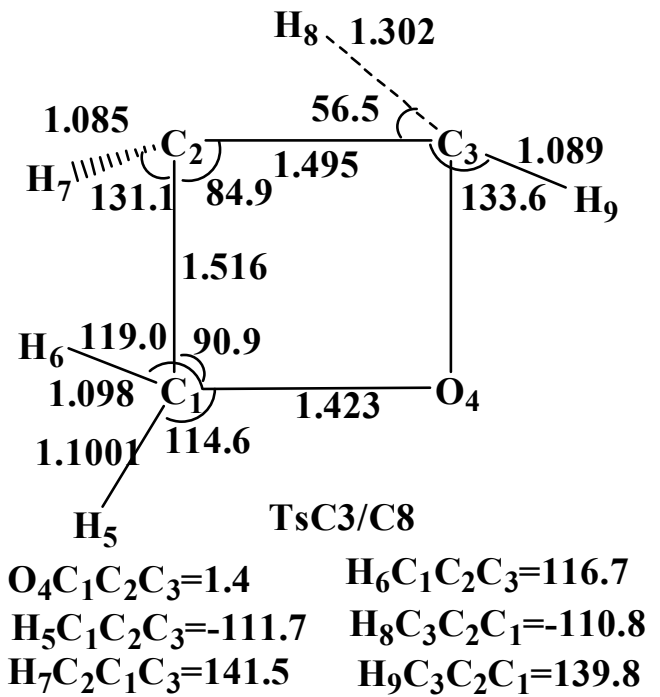

$\mathrm{H}_{7} \mathrm{C}_{2} \mathrm{C}_{1} \mathrm{C}_{3}=141.5 \quad \mathrm{H}_{9} \mathrm{C}_{3} \mathrm{C}_{2} \mathrm{C}_{1}=139.8$

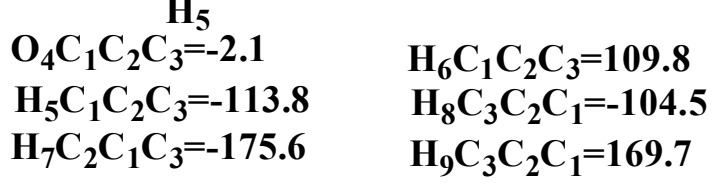

$\mathrm{O}_{4} \mathrm{C}_{3} \mathrm{C}_{2} \mathrm{C}_{1}=79.8$

$\mathrm{H}_{5} \mathrm{C}_{1} \mathrm{C}_{2} \mathrm{C}_{3}=-71.9$

$\mathrm{H}_{7} \mathrm{C}_{2} \mathrm{C}_{1} \mathrm{C}_{3}=\mathbf{1 1 7 . 0}$

${ }_{6} \mathrm{C}_{1} \mathrm{C}_{2} \mathrm{C}_{3}=88.2$

$\mathrm{H}_{8} \mathrm{C}_{2} \mathrm{C}_{1} \mathrm{C}_{3}=-121.2$

$\mathrm{H}_{9} \mathrm{O}_{4} \mathrm{C}_{3} \mathrm{C}_{2}=205.8$ 
SIFigure 1 (Continued)

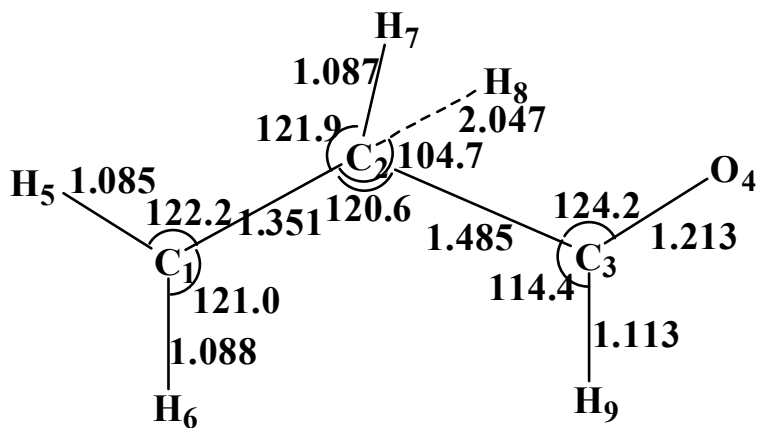

TsL3/P1

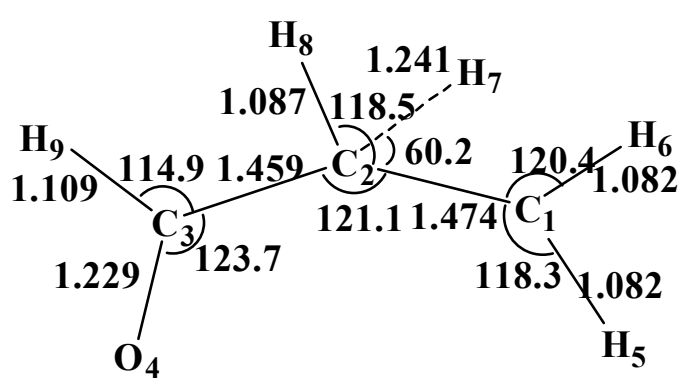

TsL3/L5

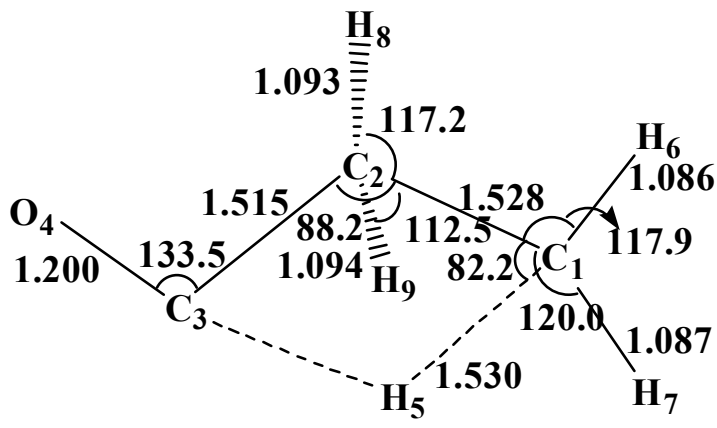

TsL3/L1
$\mathrm{H}_{6} \mathrm{C}_{1} \mathrm{C}_{2} \mathrm{C}_{3}=8.1$

$\mathrm{H}_{8} \mathrm{C}_{2} \mathrm{C}_{1} \mathrm{C}_{3}=-99.2$

$\mathrm{H}_{9} \mathrm{C}_{3} \mathrm{C}_{2} \mathrm{C}_{1}=-12.4$

$\mathrm{H}_{7} \mathrm{C}_{2} \mathrm{C}_{1} \mathrm{C}_{3}=167.2$

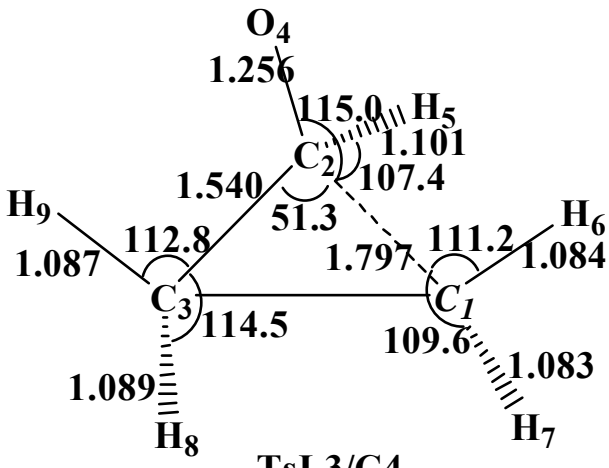

TsL3/C4
$\mathrm{O}_{4} \mathrm{C}_{2} \mathrm{C}_{1} \mathrm{C}_{3}=112.0 \quad \mathrm{H}_{6} \mathrm{C}_{1} \mathrm{C}_{2} \mathrm{C}_{3}=-113.7$

$\mathrm{H}_{5} \mathrm{C}_{2} \mathrm{C}_{1} \mathrm{C}_{3}=-108.7 \quad \mathrm{H}_{8} \mathrm{C}_{3} \mathrm{C}_{2} \mathrm{C}_{1}=-113.2$

$\mathrm{H}_{7} \mathrm{C}_{1} \mathrm{C}_{2} \mathrm{C}_{3}=114.2 \quad \mathrm{H}_{9} \mathrm{C}_{3} \mathrm{C}_{2} \mathrm{C}_{1}=113.3$

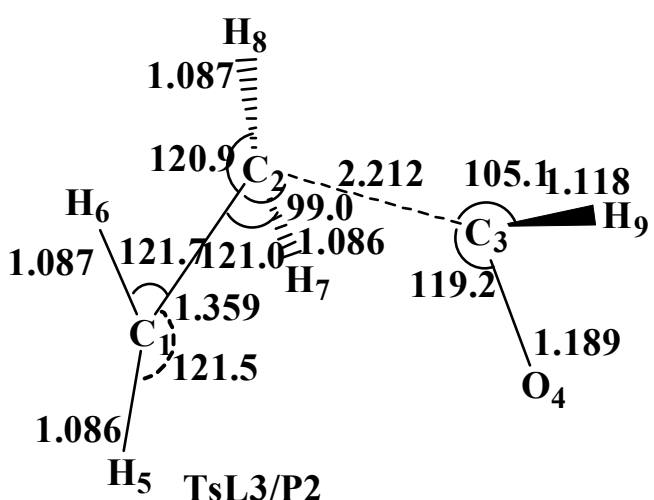

$\mathrm{O}_{4} \mathrm{C}_{3} \mathrm{C}_{2} \mathrm{C}_{1}=94.8$
$\mathrm{H}_{5} \mathrm{C}_{1} \mathrm{C}_{2} \mathrm{C}_{3}=-85.0$

$\mathrm{H}_{7} \mathrm{C}_{2} \mathrm{C}_{1} \mathrm{C}_{3}=96.3$
$\mathrm{H}_{6} \mathrm{C}_{1} \mathrm{C}_{2} \mathrm{C}_{3}=91.3$

$\mathrm{H}_{8} \mathrm{C}_{2} \mathrm{C}_{1} \mathrm{C}_{3}=-101.8$

$\mathrm{H}_{9} \mathrm{C}_{3} \mathrm{C}_{2} \mathrm{C}_{1}=-51.4$

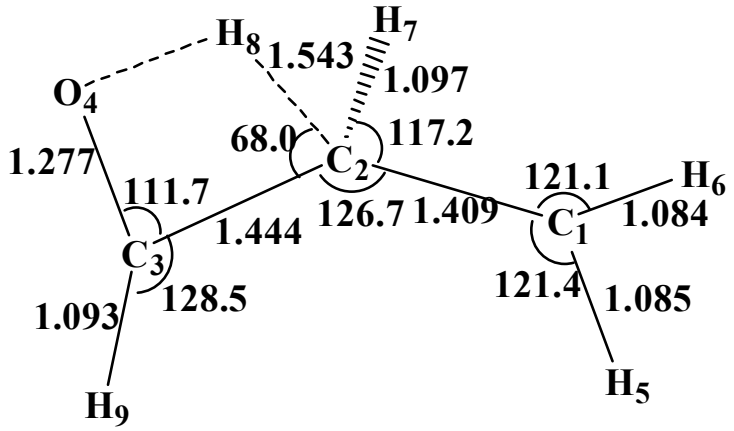

TsL3/L2

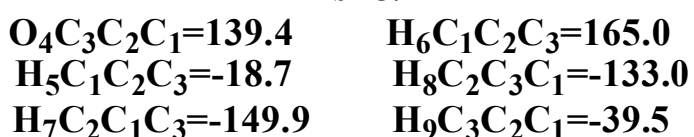

$\mathrm{H}_{7} \mathrm{C}_{2} \mathrm{C}_{1} \mathrm{C}_{3}=-149.9 \quad \mathrm{H}_{9} \mathrm{C}_{3} \mathrm{C}_{2} \mathrm{C}_{1}=-39.5$ 


\section{SIFigure 1 (Continued)}
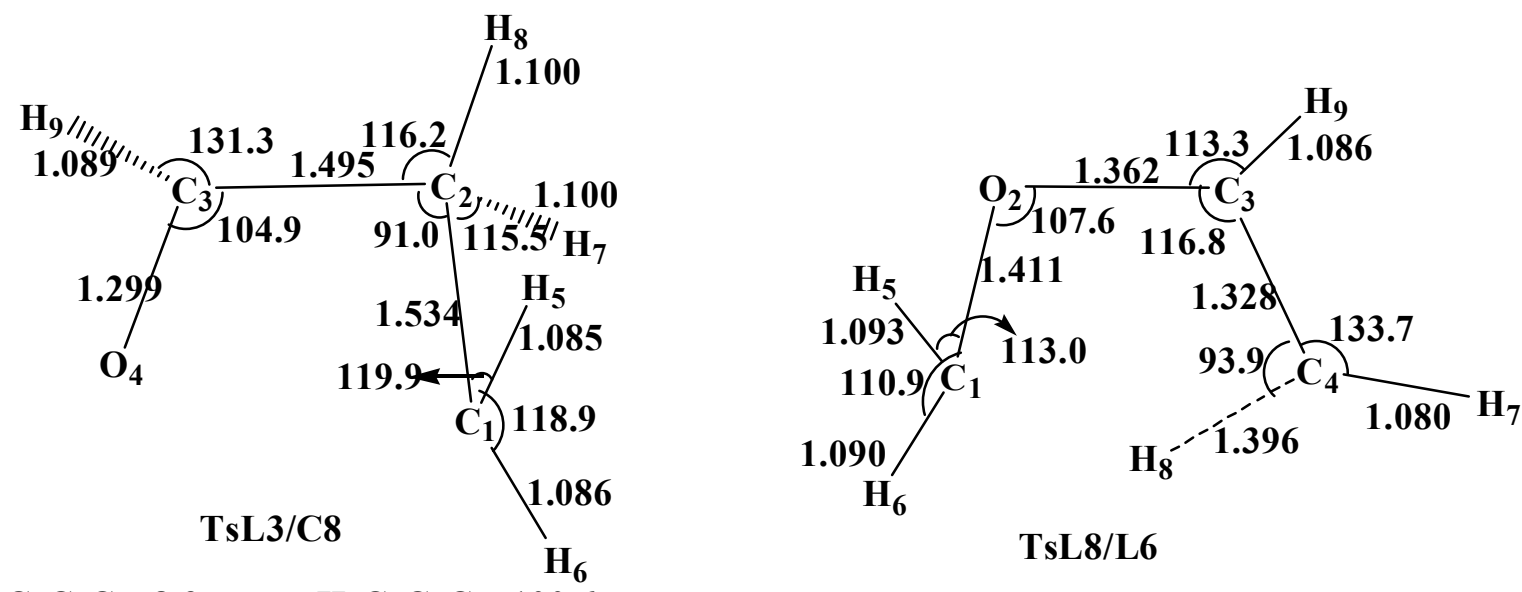

$\mathrm{O}_{4} \mathrm{C}_{3} \mathrm{C}_{2} \mathrm{C}_{1}=8.3 \quad \mathrm{H}_{6} \mathrm{C}_{1} \mathrm{C}_{2} \mathrm{C}_{3}=\mathbf{1 0 0 . 6}$

$\mathrm{H}_{5} \mathrm{C}_{1} \mathrm{C}_{2} \mathrm{C}_{3}=-.106 .9 \quad \mathrm{H}_{8} \mathrm{C}_{2} \mathrm{C}_{3} \mathrm{O}_{4}=253.3$

$\mathrm{H}_{7} \mathrm{C}_{2} \mathrm{C}_{1} \mathrm{C}_{3}=-118.4 \quad \mathrm{H}_{9} \mathrm{C}_{3} \mathrm{C}_{2} \mathrm{C}_{1}=-159.6$

TsL8/L6
$\mathrm{C}_{1} \mathrm{O}_{2} \mathrm{C}_{3} \mathrm{C}_{4}=7.4$
$\mathrm{H}_{6} \mathrm{C}_{1} \mathrm{O}_{2} \mathrm{C}_{3}=-130.7$
$\mathrm{H}_{5} \mathrm{C}_{1} \mathrm{O}_{2} \mathrm{C}_{3}=98.0$
$\mathrm{H}_{8} \mathrm{C}_{4} \mathrm{C}_{3} \mathrm{O}_{2}=0.8$
$\mathrm{H}_{7} \mathrm{C}_{4} \mathrm{C}_{3} \mathrm{O}_{2}=-179.4$
$\mathrm{H}_{9} \mathrm{C}_{3} \mathrm{O}_{2} \mathrm{C}_{1}=-174.6$

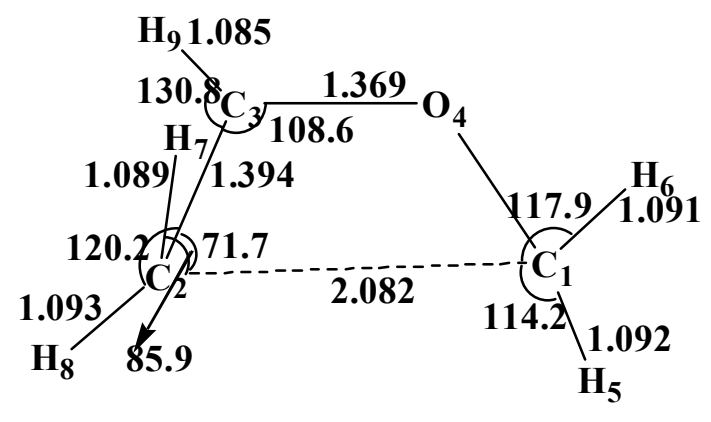

TsL8/C8

$\begin{array}{cc}\mathrm{O}_{4} \mathrm{C}_{3} \mathrm{C}_{2} \mathrm{C}_{1}=-16.4 & \mathrm{H}_{6} \mathrm{C}_{1} \mathrm{C}_{2} \mathrm{C}_{3}=125.0 \\ \mathrm{H}_{5} \mathrm{C}_{1} \mathrm{C}_{2} \mathrm{C}_{3}=-93.3 & \mathrm{H}_{8} \mathrm{C}_{2} \mathrm{C}_{3} \mathrm{C}_{4}=206.8 \\ \mathrm{H}_{7} \mathrm{C}_{2} \mathrm{C}_{1} \mathrm{O}_{4}=-108.7 & \mathrm{H}_{9} \mathrm{C}_{3} \mathrm{C}_{2} \mathrm{C}_{1}=143.5\end{array}$

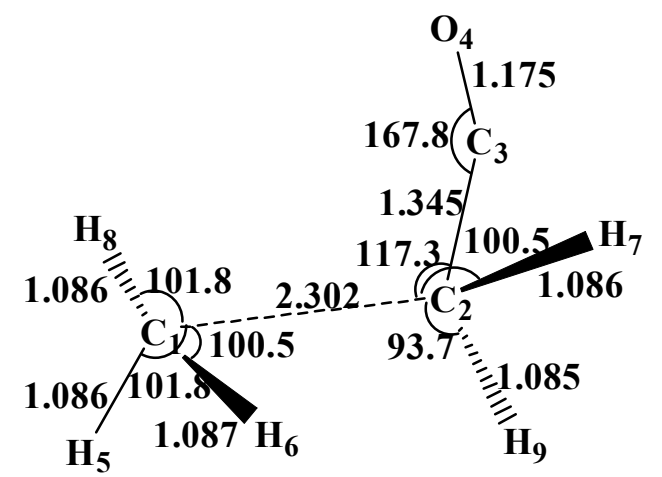

TsL1/P4

$\begin{array}{ll}\mathrm{O}_{4} \mathrm{C}_{3} \mathrm{C}_{2} \mathrm{C}_{1}=-0.0 & \mathrm{H}_{6} \mathrm{C}_{1} \mathrm{C}_{2} \mathrm{C}_{3}=180.0 \\ \mathrm{H}_{5} \mathrm{C}_{1} \mathrm{C}_{2} \mathrm{C}_{3}=60.0 & \mathrm{H}_{8} \mathrm{C}_{1} \mathrm{C}_{2} \mathrm{C}_{3}=-60.3 \\ \mathrm{H}_{7} \mathrm{C}_{2} \mathrm{C}_{1} \mathrm{C}_{3}=121.6 & \mathrm{H}_{9} \mathrm{C}_{2} \mathrm{C}_{1} \mathrm{C}_{3}=-121.6\end{array}$

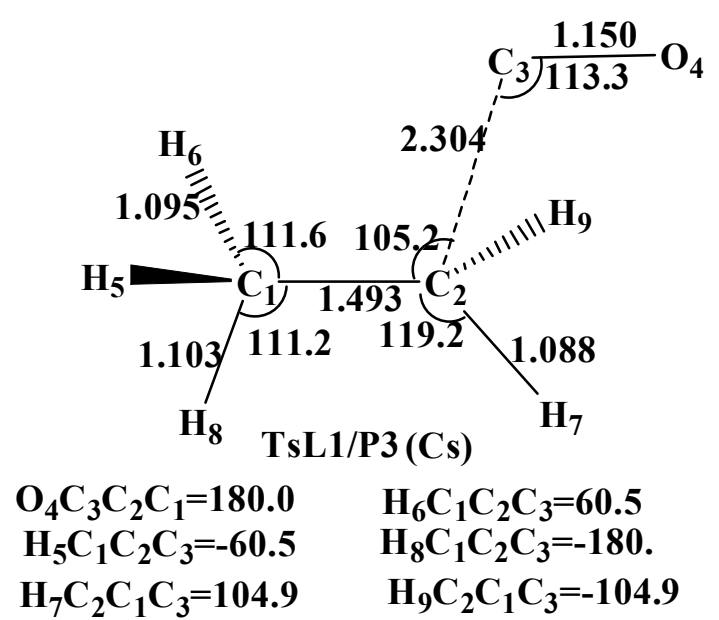


SIFigure 1 (Continued)
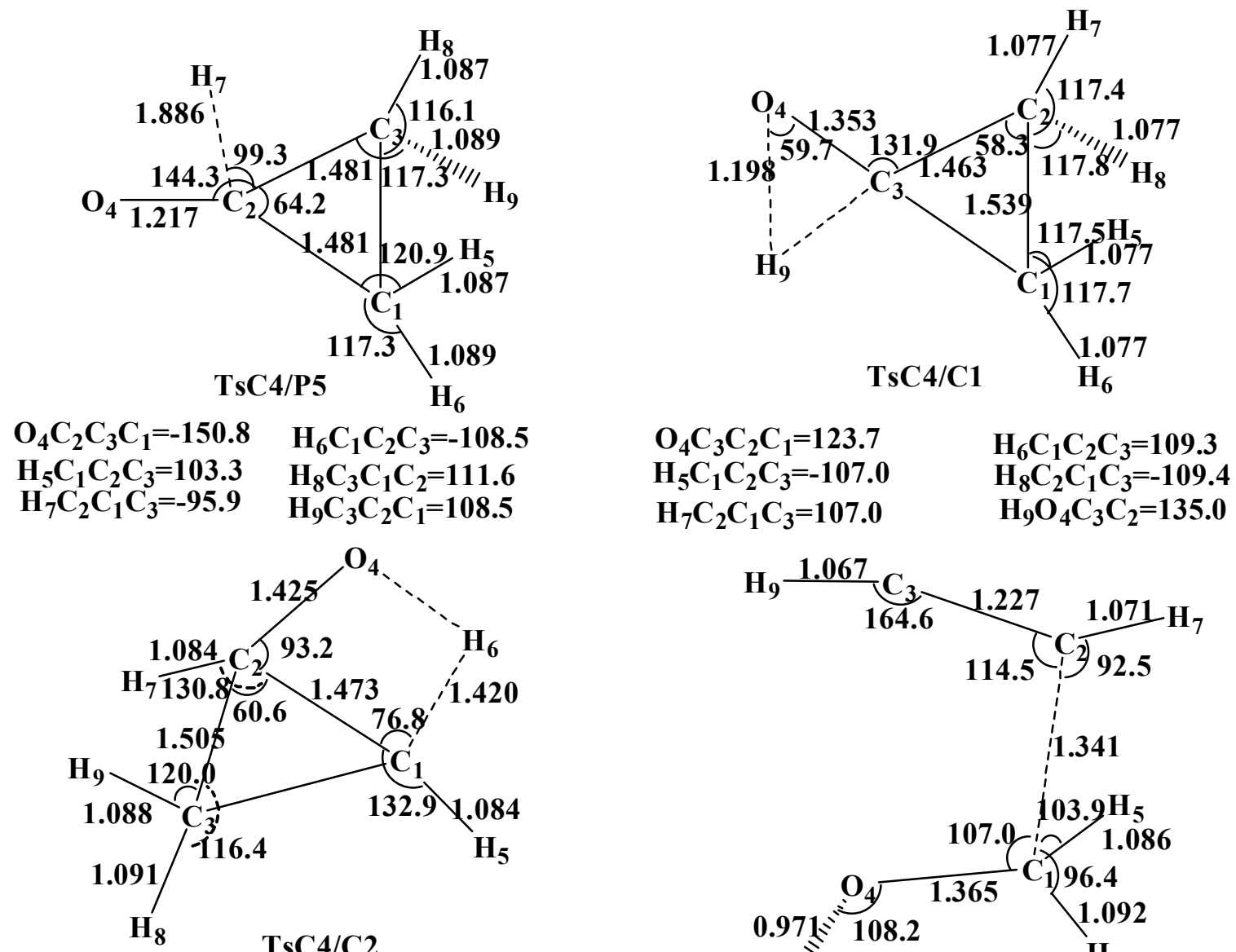

$$
\begin{array}{cc}
\mathrm{O}_{4} C_{2} C_{1} C_{3}=112.3 & H_{6} C_{1} C_{2} C_{3}=-109.4 \\
H_{5} C_{1} C_{2} C_{3}=126.9 & H_{8} C_{3} C_{2} C_{1}=-104.1 \\
H_{7} C_{2} C_{1} C_{3}=-115.3 & H_{9} C_{3} C_{2} C_{1}=110.0
\end{array}
$$
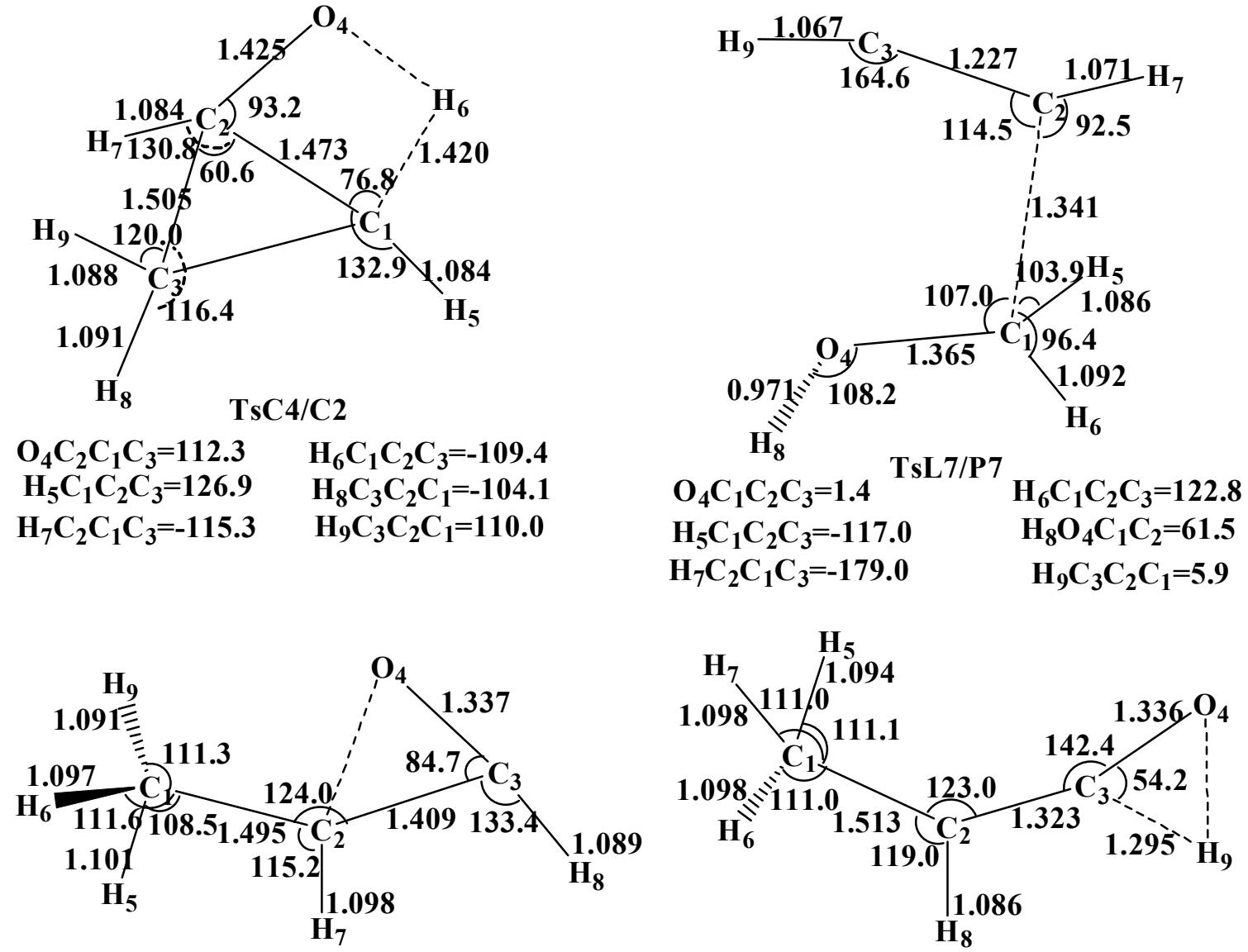

TsL5/C7
$\mathrm{O}_{4} \mathrm{C}_{3} \mathrm{C}_{2} \mathrm{C}_{1}=74.5$
$\mathrm{H}_{6} \mathrm{C}_{1} \mathrm{C}_{2} \mathrm{C}_{3}=-125.7$
$\mathrm{H}_{5} \mathrm{C}_{1} \mathrm{C}_{2} \mathrm{C}_{3}=116.9$
$\mathrm{H}_{8} \mathrm{C}_{3} \mathrm{C}_{2} \mathrm{C}_{1}=-151.2$
$\mathrm{H}_{7} \mathrm{C}_{2} \mathrm{C}_{1} \mathrm{C}_{3}=-179.8$
$\mathrm{H}_{9} \mathrm{C}_{1} \mathrm{C}_{2} \mathrm{C}_{3}=-3.0$
$\mathrm{O}_{4} \mathrm{C}_{3} \mathrm{C}_{2} \mathrm{C}_{1}=0.0 \quad \mathrm{H}_{6} \mathrm{C}_{1} \mathrm{C}_{2} \mathrm{C}_{3}=120.3$
$\mathrm{H}_{5} \mathrm{C}_{1} \mathrm{C}_{2} \mathrm{C}_{3}=0.0 \quad \mathrm{H}_{8} \mathrm{C}_{2} \mathrm{C}_{1} \mathrm{C}_{3}=-180.0$
$\mathrm{H}_{7} \mathrm{C}_{1} \mathrm{C}_{2} \mathrm{C}_{3}=-120.3 \quad \mathrm{H}_{9} \mathrm{C}_{3} \mathrm{O}_{4} \mathrm{C}_{2}=-180.0$


SIFigure 1 (Continued)

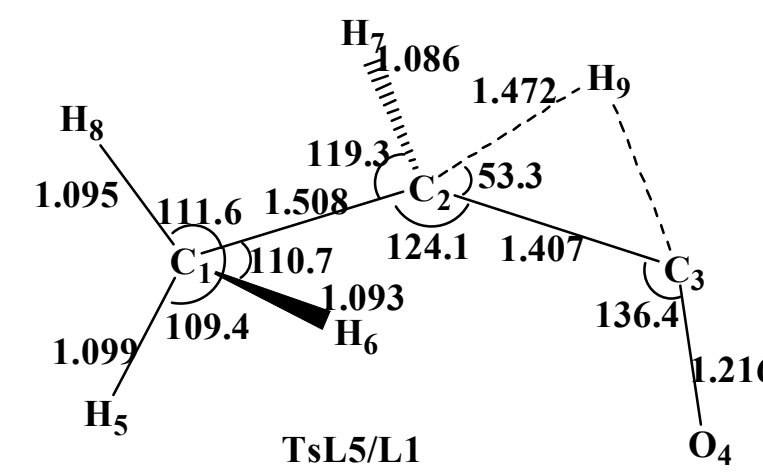

$$
\begin{array}{ll}
\mathrm{O}_{4} \mathrm{C}_{3} \mathrm{C}_{2} \mathrm{C}_{1}=0.4 & \mathrm{H}_{6} \mathrm{C}_{1} \mathrm{C}_{2} \mathrm{C}_{3}=19.7 \\
\mathrm{H}_{5} \mathrm{C}_{1} \mathrm{C}_{2} \mathrm{C}_{3}=-99.2 & \mathrm{H}_{8} \mathrm{C}_{1} \mathrm{C}_{2} \mathrm{C}_{3}=-218.2 \\
\mathrm{H}_{7} \mathrm{C}_{2} \mathrm{C}_{1} \mathrm{C}_{3}=171.3 & \mathrm{H}_{9} \mathrm{C}_{2} \mathrm{C}_{3} \mathrm{C}_{1}=118.0
\end{array}
$$

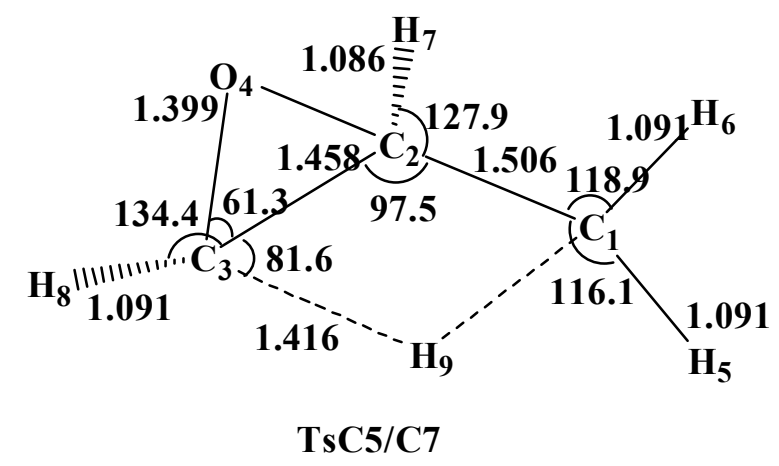

$$
\begin{array}{ll}
\mathrm{O}_{4} \mathrm{C}_{2} \mathrm{C}_{1} \mathrm{C}_{3}=108.0 & \mathrm{H}_{6} \mathrm{C}_{1} \mathrm{C}_{2} \mathrm{C}_{3}=-111.1 \\
\mathrm{H}_{5} \mathrm{C}_{1} \mathrm{C}_{2} \mathrm{C}_{3}=103.7 & \mathrm{H}_{8} \mathrm{C}_{3} \mathrm{C}_{2} \mathrm{C}_{1}=-145.3 \\
\mathrm{H}_{7} \mathrm{C}_{2} \mathrm{C}_{1} \mathrm{C}_{3}=-154.1 & \mathrm{H}_{9} \mathrm{C}_{3} \mathrm{C}_{2} \mathrm{C}_{1}=\mathbf{0 . 8 6}
\end{array}
$$

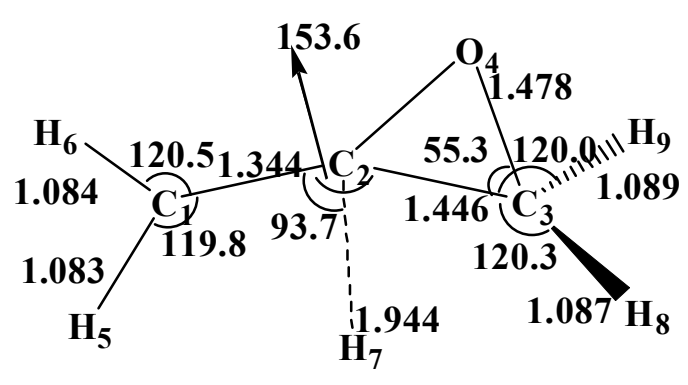

TsC5/P7

$$
\begin{array}{ll}
\mathrm{O}_{4} \mathrm{C}_{3} \mathrm{C}_{2} \mathrm{C}_{1}=145.9 & \mathrm{H}_{6} \mathrm{C}_{1} \mathrm{C}_{2} \mathrm{C}_{3}=-150.8 \\
\mathrm{H}_{5} \mathrm{C}_{1} \mathrm{C}_{2} \mathrm{C}_{3}=38.1 & \mathrm{H}_{8} \mathrm{C}_{3} \mathrm{C}_{2} \mathrm{C}_{1}=-113.8 \\
\mathrm{H}_{7} \mathrm{C}_{2} \mathrm{C}_{1} \mathrm{C}_{3}=-112.5 & \mathrm{H}_{9} \mathrm{C}_{3} \mathrm{C}_{2} \mathrm{C}_{1}=45.9
\end{array}
$$

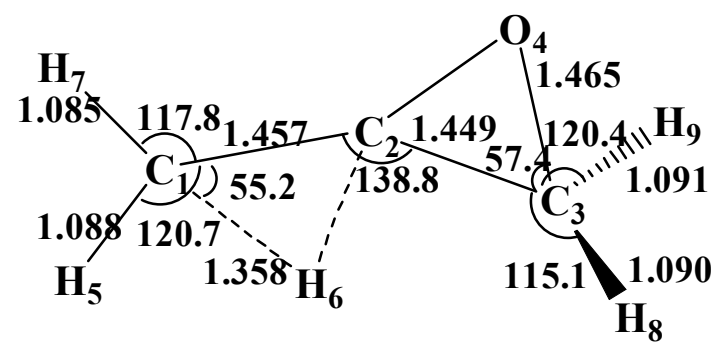

TsC5/C6

$$
\begin{array}{cc}
\mathrm{O}_{4} \mathrm{C}_{3} \mathrm{C}_{2} \mathrm{C}_{1}=123.4 & \mathrm{H}_{6} \mathrm{C}_{1} \mathrm{C}_{2} \mathrm{C}_{3}=131.1 \\
\mathrm{H}_{5} \mathrm{C}_{1} \mathrm{C}_{2} \mathrm{C}_{3}=26.7 & \mathrm{H}_{8} \mathrm{C}_{3} \mathrm{O}_{4} \mathrm{C}_{2}=-11.5 \\
\mathrm{H}_{7} \mathrm{C}_{1} \mathrm{C}_{2} \mathrm{C}_{3}=-135.8 & \mathrm{H}_{9} \mathrm{C}_{3} \mathrm{C}_{2} \mathrm{C}_{1}=22.9
\end{array}
$$

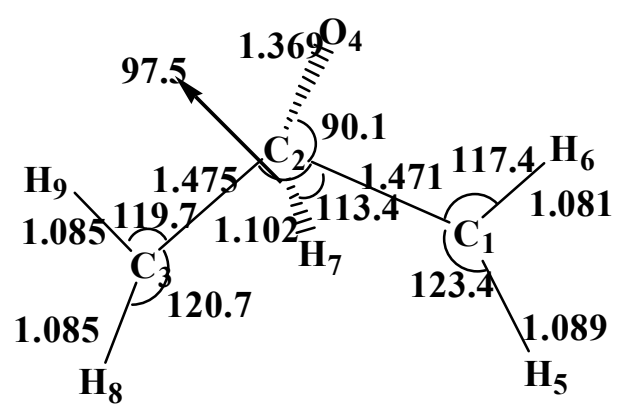

TsC5/C4

$\begin{array}{ll}\mathrm{O}_{4} C_{2} C_{1} C_{3}=122.9 & H_{6} C_{1} C_{2} C_{3}=-60.4 \\ H_{5} C_{1} C_{2} C_{3}=125.0 & H_{8} C_{3} C_{2} C_{1}=-101.1 \\ H_{7} C_{1} C_{2} C_{3}=-118.9 & H_{9} C_{3} C_{2} C_{1}=72.3\end{array}$

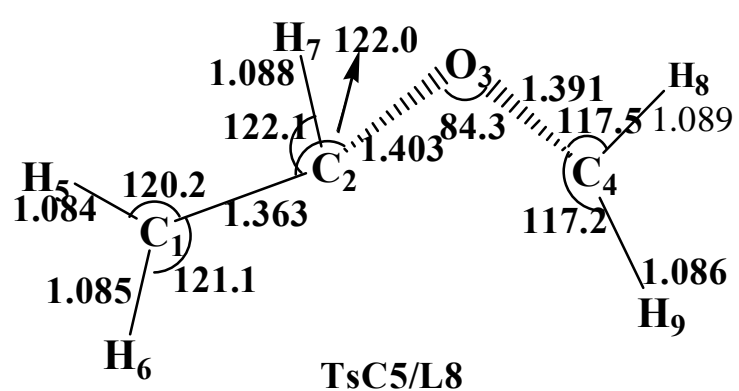

$$
\begin{array}{ll}
\mathrm{C}_{4} \mathrm{O}_{3} \mathrm{C}_{2} \mathrm{C}_{1}=-106.2 & \mathrm{H}_{6} \mathrm{C}_{1} \mathrm{C}_{2} \mathrm{C}_{3}=6.1 \\
\mathrm{H}_{5} \mathrm{C}_{1} \mathrm{C}_{2} \mathrm{C}_{3}=-76.0 & \mathrm{H}_{8} \mathrm{C}_{3} \mathrm{O}_{4} \mathrm{C}_{2}=-3.2 \\
\mathrm{H}_{7} \mathrm{C}_{2} \mathrm{C}_{1} \mathrm{C}_{3}=167.2 & \mathrm{H}_{9} \mathrm{C}_{3} \mathrm{C}_{2} \mathrm{C}_{1}=99.4
\end{array}
$$


SITable 1

\begin{tabular}{|c|c|c|c|}
\hline Species & G3B3 & Species & G3B3 \\
\hline $\mathrm{R} \mathrm{C}_{2} \mathrm{H}_{3}+\mathrm{H}_{2} \mathrm{CO}$ & $\begin{array}{l}-192.26895(0 .) \\
-192.08712(0.0)^{b}\end{array}$ & TsR/P2 & $\begin{array}{c}-192.26153(4.7) \\
-192.08097(3.9)\end{array}$ \\
\hline $\mathrm{LO} \mathrm{CH}_{2} \mathrm{CH}_{2} \mathrm{COH}$ & -192.24681 (13.9) & TsR/L8 & $-192.24620(14.3)$ \\
\hline $\mathrm{L} 1 \mathrm{CH}_{3} \mathrm{CH}_{2} \mathrm{CO}$ & $-192.34789(-49.5)$ & TsR/P7 & $-192.21557(33.5)$ \\
\hline $\mathrm{L} 2 \mathrm{CH}_{2} \mathrm{CHCHOH}$ & $-192.33755(-43.1)$ & TsR/C8 & $192.15622(70.7)$ \\
\hline $\mathrm{L} 3 \mathrm{CH}_{2} \mathrm{CH}_{2} \mathrm{CHO}$ & $-192.32824(-37.2)$ & TsR/L10 & $\begin{array}{l}-192.26447(2.8) \\
-192.08345(2.3)\end{array}$ \\
\hline $\mathrm{L} 4 \mathrm{CH}_{3} \mathrm{CHCOH}$ & $-192.30284(-21.2)$ & TsL10/C5 & $-192.28792(-11.9)$ \\
\hline $\mathrm{L} 5 \mathrm{CH}_{3} \mathrm{CHCHO}$ & $-192.34742(-49.2)$ & TsL10/C3 & $-192.25248(10.3)$ \\
\hline $\mathrm{L}_{6} \mathrm{CHCHOCH}_{3}$ & $-192.28140(-7.8)$ & TsL10/P1 & $\begin{array}{l}-192.27136(-1.5) \\
-192.09037(-2.0)\end{array}$ \\
\hline $\mathrm{L} 7 \mathrm{CHCHCH}_{2} \mathrm{OH}$ & $-192.29072(-13.7)$ & TsL10/L2 & $-192.26241(4.1)$ \\
\hline $\mathrm{L} 8 \mathrm{CH}_{2} \mathrm{CHOCH}_{2}$ & $-192.303885(-21.9)$ & TsL10/L3 & $\begin{array}{l}-192.27037(-0.9) \\
-192.09104(-2.5)\end{array}$ \\
\hline $\mathrm{L} 9 \mathrm{CH}_{2} \mathrm{CCH}_{2} \mathrm{OH}$ & $-192.29229(-14.6)$ & TsL10/L7 & $-192.26053(5.3)$ \\
\hline $\mathrm{L} 10 \mathrm{CH}_{2} \mathrm{CHCH}_{2} \mathrm{O}$ & $-192.30060(-37.2)$ & TsL10/L5 & $-192.18389(53.4)$ \\
\hline $\mathrm{C} 1 \mathrm{OH}-\mathrm{CCCH}_{2} \mathrm{CH}_{2}$ & $-192.29283(-15.0)$ & TsC3/P6 & $-192.23008(24.4)$ \\
\hline $\mathrm{C} 2 \mathrm{OH}-\mathrm{CCHCH}_{2} \mathrm{CH}$ & $-192.28552(-10.4)$ & TsC3/C8 & $-192.21811(31.9)$ \\
\hline $\mathrm{C} 4 \mathrm{O}-\mathrm{cCHCH}_{2} \mathrm{CH}_{2}$ & $-192.30811(-24.6)$ & TsL3/P1 & -192.27585 (4.33) \\
\hline $\mathrm{C} 3 \mathrm{cCH}_{2} \mathrm{CHCH}_{2} \mathrm{O}$ & $-192.28750(-11.6)$ & TsL3/C4 & $-192.30918(-25.2)$ \\
\hline $\mathrm{C} 5 \mathrm{H}_{2} \mathrm{C}-\mathrm{cCOCH} \mathrm{CH}_{2}$ & $-192.29636(-17.2)$ & TsL3/C8 & $-192.25533(8.5)$ \\
\hline $\mathrm{C} 6 \mathrm{CH}_{3}-\mathrm{CCCH}_{2} \mathrm{O}$ & $-192.29149(-14.2)$ & TsL3/L1 & $-192.27234(-2.1)$ \\
\hline $\mathrm{C} 7 \mathrm{CH}_{3}-\mathrm{CCHCHO}$ & $-192.28932(-12.8)$ & TsL3/L0 & $-192.20205(42.0)$ \\
\hline $\mathrm{C} 8 \mathrm{cCH}_{2} \mathrm{CHOCH}_{2}$ & $-192.29718(-17.7)$ & TsL3/L2 & $-192.24061(17.8)$ \\
\hline $\mathrm{P} 2 \mathrm{CH}_{2} \mathrm{CH}_{2}+\mathrm{CHO}$ & $-192.30339(-21.6)$ & TsL8/L6 & -192.25481 (8.9) \\
\hline $\mathrm{P} 3 \mathrm{CO}+\mathrm{CH}_{2} \mathrm{CH}_{3}$ & $-192.33566(-41.9)$ & TsL8/C8 & -192.24378 (15.8) \\
\hline $\mathrm{P} 4 \mathrm{CH}_{3}+\mathrm{CH}_{2} \mathrm{CO}$ & $-192.29150(-14.1)$ & TsL7/P7 & $-192.24514(14.9)$ \\
\hline $\mathrm{P} 7 \mathrm{CHCH}+\mathrm{CH}_{2} \mathrm{OH}$ & $-192.25830(6.7)$ & TsL2/C2 & -192.26318 (3.6) \\
\hline $\mathrm{P} 1 \mathrm{CH}_{2} \mathrm{CHCHO}+\mathrm{H}$ & $-192.28085(-7.5)$ & TsL2/P1 & $-192.26789(0.7)$ \\
\hline $\mathrm{P} 8 \mathrm{CH}_{2}-\mathrm{CCCH}_{2} \mathrm{O}+\mathrm{H}$ & $-192.23180(23.3)$ & TsC4/P5 & $-192.23917(18.7)$ \\
\hline $\mathrm{P} 5 \mathrm{cCH}_{2} \mathrm{CH}_{2} \mathrm{CO}+\mathrm{H}$ & -192.24767(13.4) & TsC4/C2 & $-192.22750(26.0)$ \\
\hline $\mathrm{P} 6 \mathrm{cCH}_{2} \mathrm{CHCHO}+\mathrm{H}$ & $-192.23417(21.8)$ & TsC4/C1 & $-192.23130(23.6)$ \\
\hline TsC5/C6 & $-192.21377(34.6)$ & TsL1/P3 & $-192.32651(-36.1)$ \\
\hline TsC5/P7 & $-192.22404(28.2)$ & TsL1/P4 & $-192.28445(-9.7)$ \\
\hline TsC5/C4 & -192.22137 (29.9) & TsL1/L0 & $-192.23619(20.6)$ \\
\hline TsC5/C7 & $-192.21707(32.56)$ & TsL5/L1 & $-192.28011(-7.0)$ \\
\hline TsC5/L8 & $-192.27613(-4.5)$ & TsL5/L4 & $-192.24915(12.4)$ \\
\hline TsL3/P2 & $-192.29164(-14.2)$ & TsL5/C7 & $-192.26404(3.1)$ \\
\hline TsL3/L5 & $-192.28254(-8.53)$ & TsL2/L9 & $-192.22854(25.4)$ \\
\hline
\end{tabular}

